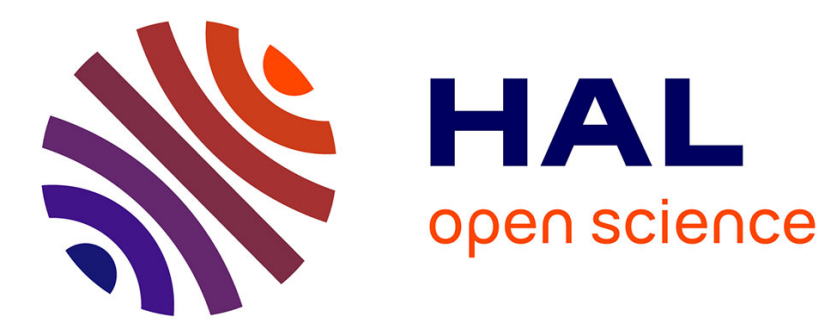

\title{
On the Use of First-order Autoregressive Modeling for Rayleigh Flat Fading Channel Estimation with Kalman Filter
}

Soukayna Ghandour - Haidar, Laurent Ros, Jean-Marc Brossier

\section{- To cite this version:}

Soukayna Ghandour - Haidar, Laurent Ros, Jean-Marc Brossier. On the Use of First-order Autoregressive Modeling for Rayleigh Flat Fading Channel Estimation with Kalman Filter. Signal Processing, 2012, 92 (2), pp.601-606. 10.1016/j.sigpro.2011.08.014 . hal-00638787

\section{HAL Id: hal-00638787 \\ https://hal.science/hal-00638787}

Submitted on 7 Nov 2011

HAL is a multi-disciplinary open access archive for the deposit and dissemination of scientific research documents, whether they are published or not. The documents may come from teaching and research institutions in France or abroad, or from public or private research centers.
L'archive ouverte pluridisciplinaire HAL, est destinée au dépôt et à la diffusion de documents scientifiques de niveau recherche, publiés ou non, émanant des établissements d'enseignement et de recherche français ou étrangers, des laboratoires publics ou privés. 


\title{
On the Use of First-order Autoregressive Modeling for Rayleigh Flat Fading Channel Estimation with Kalman
}

\section{Filter}

\author{
Soukayna Ghandour-Haidar*, Laurent Ros, Jean-Marc Brossier \\ The authors are with Grenoble University (Institut Polytechnique), GIPSA-Lab, BP46, 38402 Saint-Martin \\ d'Hères, France, (email: soukayna.ghandour@gipsa-lab.grenoble-inp.fr, \\ laurent.ros@gipsa-lab.grenoble-inp.fr,jean-marc.brossier@gipsa-lab.grenoble-inp.fr).
}

\begin{abstract}
This letter deals with the estimation of a flat fading Rayleigh channel with Jakes's spectrum. The channel is approximated by a first-order autoregressive $(A R(1))$ model and tracked by a Kalman Filter (KF). The common method used in the literature to estimate the parameter of the $A R(1)$ model is based on a Correlation Matching (CM) criterion. However, for slow fading variations, another criterion based on the Minimization of the Asymptotic Variance (MAV) of the KF is more appropriate, as already observed in few works [1]. This letter gives analytic justification by providing approximated closed-form expressions of the estimation variance for the CM and MAV criteria, and of the optimal AR(1) parameter.
\end{abstract}

Keywords: Channel estimation, Autoregressive model, Kalman Filter, Jakes's spectrum, Rayleigh channel, Flat fading, Bayesian Cramér-Rao Bounds BCRB.

\section{Introduction}

The Rayleigh fading channel model with Jakes's Doppler spectrum is the most accepted random model to represent temporal variations (fading) of the equivalent baseband channel complex gain (CG) in wireless communication. However, this model is not always directly convenient for highly important tasks such as channel estimation or equalization. Usually, the autoregressive model AR(p) with some Gaussian assumptions (most often used with order $p=1$ ) is used to approach this channel and facilitate its manipulation. In [2], the authors demonstrated that the AR model can be considered for the computer simulation of correlated fading channels insisting that low orders are appropriate for narrowband Doppler fading processes. Moreover, [3] showed that a first-order model is enough to capture most of the channel tap dynamics. This approximation has been widely used to track the true Jakes's spectrum channel by a Kalman Filter (KF) in various wireless communication systems ([4, 5, 6, 7, 8, 9, 10, 11],

*This letter is dedicated to my husband Jamal for his encouragement and support. 
...). This letter deals with the choice of the AR(1) coefficient. In many papers, e.g., the aforementioned works, the AR(1) coefficient calculation for a given normalized Doppler frequency $\left(f_{d} T\right)$ is based on the same criterion, called Correlation Matching (CM) in this letter. The CM criterion ${ }^{1}$ consists in imposing that the autocorrelation coefficients $R_{\tilde{\alpha}}[n]$ of the approximated $\mathrm{AR}(1)$ process $\tilde{\alpha}$ perfectly match the sampled autocorrelation function (which is a Bessel function) of the true CG $\alpha$ for lags $n \in\{-1,0,1\}$ [2] (see also [4], Fig. 1). But in some situations the results were disappointing (in terms of the distance from the Bayesian Cramér-Rao lower Bound (BCRB)), as pointed out recently in [10] (Fig. 3) and in [11] (Fig. 2) for the special case of a slow fading scenario where the channel is theoretically easier to estimate. Note also that some authors propose adding a positive $\varepsilon$ to the zeroth autocorrelation lag, which modifies slightly the CM constraint to better approximate the original process in some sense [2]. In the recent work [1], the search for the AR(1) parameter is based on another method, consisting in the minimization of the steady-state estimation error variance, called the Minimum Asymptotic Variance (MAV) criterion in this letter. This method seems very effective compared to the CM method for a Clarke (i.e., Rayleigh-Jakes) Model channel, but the study in [1] does not provide analytic results nor closed-form expressions about the choice of the AR(1) parameter (nor the associated Mean Square Error (MSE)) for a given channel state ( $f_{d} T$, SNR). In this letter, we first provide a general theoretical frequency-domain analysis of the estimation error (in terms of static and dynamic contributions) that explains analytically the previous disappointing results with the CM-criterion based method. Moreover, the analytic approach is used to calculate the optimal $\mathrm{AR}(1)$ coefficient under the MAV criterion, without the CM constraint. For a given Doppler and SNR scenario, we give approximate closedform expressions of the optimal coefficient and of the corresponding MSE.

\section{Mathematical Model}

We consider the estimation of a flat Rayleigh fading channel. The discrete-time observation is ${ }^{2}$ :

$$
y_{k}=\alpha_{k}+n_{k}
$$

where $k$ is the (symbol) time index, $n_{k}$ is a zero-mean additive white circular complex Gaussian noise with variance $\sigma_{n}^{2}$, and $\alpha_{k}$ is a zero-mean circular complex Gaussian CG with variance $\sigma_{\alpha}^{2}=1$. The normalized Doppler frequency of this channel is $f_{d} T$, where

\footnotetext{
${ }^{1}$ And for a more general AR(p) model (with more degrees of freedom than the AR(1) model), the coefficients of the $\mathrm{AR}(\mathrm{p})$ process are calculated [2] by still imposing the correlation matching constraint for lags $n \in\{-p, \ldots,-1,0,1, \ldots, p\}$, but followed by the resolution of the Yule-Walker equations in order to minimize the prediction error (model noise).

${ }^{2}$ In general, the observation is rather $y_{k}=s_{k} \alpha_{k}+n_{k}$ where $s_{k}$ denotes the transmitted symbols. Our simplified model (1) assumes the symbols are known (or decided). This model relies also on the assumption that the $\mathrm{CG}$ variation can be neglected during one symbol period. It should be noted that even if it is simple, this observation model is the same as the one obtained through multipath frequency selective channel in an OFDM system, for each subcarrier.
} 
$T$ is the symbol period. A Jakes's Doppler spectrum is assumed for this channel:

$$
\Gamma_{\alpha}(f)= \begin{cases}\frac{\sigma_{\alpha}^{2}}{\pi f_{d} \sqrt{1-\left(\frac{f}{f_{d}}\right)^{2}}} & \text { if }|f|<f_{d} \\ 0 & \text { if }|f|>f_{d}\end{cases}
$$

The autocorrelation matrix $\boldsymbol{R}_{\boldsymbol{\alpha}}^{k}$ of a block of $k$ consecutive CG is then defined by its $[i, j]$ th entry:

$$
\left[\boldsymbol{R}_{\alpha}^{k}\right]_{i, j}=R_{\alpha}[i-j]=\sigma_{\alpha}^{2} J_{0}\left(2 \pi f_{d} T .(i-j)\right) \quad 1 \leq i, j \leq k
$$

where $J_{0}$ is the zeroth-order Bessel function of the first kind.

The time-varying CG $\alpha_{k}$ is approached by a first-order autoregressive $(\operatorname{AR}(1))$ model $\tilde{\alpha}_{k}$ :

$$
\tilde{\alpha}_{k}=a \cdot \tilde{\alpha}_{k-1}+e_{k}
$$

where $e_{k}$ is a white circular complex Gaussian noise with variance $\sigma_{e}^{2}=\left(1-a^{2}\right) \sigma_{\alpha}^{2}$. The observation equation (4) is then approximated by:

$$
y_{k}=\tilde{\alpha}_{k}+n_{k}
$$

The AR(1) coefficient verifies

$$
a=\frac{R_{\tilde{\alpha}}[1]}{R_{\tilde{\alpha}}[0]}
$$

If the correlation matching (CM) criterion $[2,4,5,6,8]$ is imposed, the two first autocorrelation coefficients of the $\operatorname{AR}(1)$ process $\tilde{\alpha}$ coincide with those of the exact CG $\alpha$ (i.e., $R_{\tilde{\alpha}}[0]=R_{\alpha}[0]$, and $R_{\tilde{\alpha}}[1]=R_{\alpha}[1]$ ), and using equation (6) and (3) the $\operatorname{AR}(1)$ coefficient $a$ noted $a_{C M}$ becomes

$$
a_{C M}=J_{0}\left(2 \pi f_{d} T\right)
$$

\section{MSE Analysis, Optimization, and Simulation Results}

Given the model and observation equations ( 4 and 5), we use a KF to get an on-line unbiased estimate, $\hat{\alpha}_{k}$, of the true $\alpha_{k}$. We compare the variance of the error $\varepsilon_{k}=\alpha_{k}-\hat{\alpha}_{k}$ to the on-line BCRB (see [12] for details about the computation of this BCRB):

$$
E\left\{\left|\varepsilon_{k}\right|^{2}\right\} \geq B C R B(k)
$$

with $B C R B(k)=\left[\boldsymbol{J}^{-1}\right]_{k, k}{ }^{3}$ the last element of the inverse of the Bayesian Information Matrix $\boldsymbol{J}$ :

$$
\boldsymbol{J}=\frac{1}{\sigma_{n}^{2}} \boldsymbol{I}_{k}+\left[\boldsymbol{R}_{\boldsymbol{\alpha}}^{k}\right]^{-1}
$$

\footnotetext{
${ }^{3}$ The Cramér-Rao inequality is verified at every iteration $k$ but the BCRB converges to a constant value after a certain number of iterations ([12], [15]), (i.e., BCRB $\approx \mathrm{BCRB}(k)$ for a sufficiently large value of $k$ ).
} 
and $\boldsymbol{I}_{k}$ is the $k \times k$ identity matrix.

The KF is given by [14] (see also p. 436 in [16]):

$\begin{array}{ll}\text { Prediction } & \hat{\alpha}_{k \mid k-1}=a \hat{\alpha}_{k-1} \\ \text { Prediction MSE } & P_{k \mid k-1}=a^{2} P_{k-1}+\sigma_{e}^{2} \\ \text { Kalman Gain } & K_{k}=\frac{P_{k \mid k-1}}{\sigma_{n}^{2}+P_{k \mid k-1}} \\ \text { Correction } & \hat{\alpha}_{k}=\hat{\alpha}_{k \mid k-1}+K_{k}\left(y_{k}-\hat{\alpha}_{k \mid k-1}\right) \\ \text { MSE } & P_{k}=\left(1-K_{k}\right) P_{k \mid k-1}\end{array}$

Replacing (ii) in (iii), (v) in (ii), and (i) in (iv), we obtain the simplified system:

$$
\begin{aligned}
K_{k} & =\left[a^{2} P_{k-1}+\sigma_{e}^{2}\right] /\left[a^{2} P_{k-1}+\sigma_{e}^{2}+\sigma_{n}^{2}\right] \\
P_{k} & =\left(1-K_{k}\right)\left(a^{2} P_{k-1}+\sigma_{e}^{2}\right) \\
\hat{\alpha}_{k} & =a \hat{\alpha}_{k-1}+K_{k}\left(y_{k}-a \hat{\alpha}_{k-1}\right)
\end{aligned}
$$

where $K_{k}$ is the Kalman gain at iteration $k$ and $P_{k}$ is the estimation error variance. Since the linear system (1) and (4) is observable and controllable, an asymptotic regime for which $K_{k}=K_{k+1}=K_{\infty}$ and $P_{k}=P_{k+1}=P_{\infty}$ is quickly reached ([14]).

Based on equations (8) and (9), and given $a^{2}=1-\frac{\sigma_{e}^{2}}{\sigma_{\alpha}^{2}}$, we deduce

$$
\begin{aligned}
P_{\infty} & =\frac{\sigma_{n}^{2}\left(a^{2}-1\right)-\sigma_{e}^{2}+\sqrt{\Delta}}{2 a^{2}} \\
K_{\infty} & =\frac{a^{2} P_{\infty}+\sigma_{e}^{2}}{a^{2} P_{\infty}+\sigma_{e}^{2}+\sigma_{n}^{2}}
\end{aligned}
$$

with $\Delta=\left(\sigma_{n}^{2}+\sigma_{e}^{2}-a^{2} \sigma_{n}^{2}\right)^{2}+4 a^{2} \sigma_{n}^{2} \sigma_{e}^{2}$. From now on, we assume that the noise model variance satisfies $\sigma_{e}^{2}<<\sigma_{n}^{2}$. Note that this assumption means that $K_{\infty}<<1$, which seems reasonable for low normalized Doppler (i.e., $f_{d} T \leq 10^{-2}$ ) and the usual range of SNR (i.e., $0.01 \leq \frac{\sigma_{n}^{2}}{\sigma_{\alpha}^{2}} \leq 1$ ). We then have the following approximations (see Appendix A):

$$
\sqrt{\Delta} \approx 2 \sigma_{e} \sigma_{n} \quad \text { and } \quad a^{2} P_{\infty} \approx \sigma_{e} \sigma_{n}
$$

leading to the following approximate expression of the steady-state Kalman gain:

$$
K_{\infty} \approx \frac{\sigma_{e}}{\sigma_{n}}
$$

On the other hand, we see that in the asymptotic regime (or tracking mode), equation (10) of the KF reduces (using (1)) to a time-invariant linear filter, expressed in the Z-Transform domain by:

$$
\hat{\alpha}(z)=a z^{-1} \hat{\alpha}(z)+K_{\infty}\left(\alpha(z)+N(z)-a z^{-1} \hat{\alpha}(z)\right)
$$

Then $\hat{\alpha}(z)=T(z)(\alpha(z)+N(z))$ and the error is

$$
\varepsilon(z)=\alpha(z)-\hat{\alpha}(z)=(1-T(z)) \alpha(z)-T(z) N(z)
$$


where $T(z)$ is the transfer function of the steady-state KF:

$$
T(z)=\frac{K_{\infty}}{1-a\left(1-K_{\infty}\right) z^{-1}}
$$

Using (14), the asymptotic MSE is divided into two parts:

$$
M S E=M S E 1+M S E 2
$$

- MSE1 is the dynamic error variance, due to the CG variations $\alpha_{k}$ filtered by the high pass filter $1-T(z)$ :

$$
M S E 1 \stackrel{\text { def }}{=} \int_{-\frac{1}{2 T}}^{+\frac{1}{2 T}}\left|1-T\left(e^{j 2 \pi f T}\right)\right|^{2} \Gamma_{\alpha}(f) d f
$$

Under the assumption $\frac{1}{2} \frac{\sigma_{e}^{2}}{\sigma_{\alpha}^{2}}<<2 \pi f_{d} T<2 \pi f_{c} T<<1$, which is reasonable for a low-Doppler scenario, we obtain (see Appendix B):

$$
M S E 1 \approx \int_{-f_{d}}^{+f_{d}}\left|\frac{2 \pi f T}{2 \pi f_{c} T}\right|^{2} \Gamma_{\alpha}(f) d f=\sigma_{\alpha}^{2} \frac{\left(2 \pi f_{d} T\right)^{2}}{2\left(2 \pi f_{c} T\right)^{2}}
$$

where $f_{c}$ is the cut-off frequency of the low-pass filter $\mathrm{T}(\mathrm{z})$, defined such that $2 \pi f_{c} T=\frac{1-a\left(1-K_{\infty}\right)}{a\left(1-K_{\infty}\right)}$. Still assuming that $\sigma_{e}^{2}<<\sigma_{n}^{2}$, it results that $2 \pi f_{c} T \approx$ $\frac{\sigma_{n}-a\left(\sigma_{n}-\sigma_{e}\right)}{a\left(\sigma_{n}-\sigma_{e}\right)} \approx \frac{\sigma_{e}}{\sigma_{n}}$, which means that the (normalized) cut-off pulsation is approximately equal to the steady-state Kalman gain. The approximate closed-form expression of $M S E 1$ is then

$$
M S E 1 \approx \sigma_{\alpha}^{2} \frac{\left(2 \pi f_{d} T\right)^{2} \sigma_{n}^{2}}{2 \sigma_{e}^{2}}=\frac{\left(2 \pi f_{d} T\right)^{2} \sigma_{n}^{2}}{2\left(1-a^{2}\right)}
$$

- MSE2 is the static error variance, due to the additive noise $n_{k}$ filtered by the low pass filter $-T(z)$ :

$$
M S E 2 \stackrel{\text { def }}{=} \sigma_{n}^{2} T \int_{-\frac{1}{2 T}}^{+\frac{1}{2 T}}\left|T\left(e^{j 2 \pi f T}\right)\right|^{2} d f=\sigma_{n}^{2} \frac{K_{\infty}^{2}}{\left|1-a^{2}\left(1-K_{\infty}\right)^{2}\right|}
$$

Inserting (13) in (20), using again $a^{2}=1-\frac{\sigma_{e}^{2}}{\sigma_{\alpha}^{2}}$ and the previous assumption $\sigma_{e}^{2}<<\sigma_{n}^{2}$, it follows that

$$
M S E 2 \approx \sigma_{n}^{2} \cdot \frac{\sigma_{e}}{2 \sigma_{n}}=\frac{\sqrt{\left(1-a^{2}\right)} \sigma_{n} \sigma_{\alpha}}{2}
$$

These approximate closed-form expressions (19) and (21) will be useful in the perspective of an optimization (MAV criterion). But first we apply them to the case of the $\mathrm{CM}$ criterion in order to explain the performance of CM-based methods. 
By imposing the CM criterion (7), and using $\left[J_{0}(\varepsilon)\right]^{2} \approx 1-\frac{\varepsilon^{2}}{2}$ for $\varepsilon<<1$, we have $\left(1-a^{2}\right) \approx 2\left(\pi f_{d} T\right)^{2}$ and then

$$
\begin{aligned}
M S E 1_{C M} & \approx \sigma_{n}^{2} \\
M S E 2_{C M} & \approx \frac{\pi f_{d} T \sigma_{n} \sigma_{\alpha}}{\sqrt{2}}
\end{aligned}
$$

Note that these approximate closed-form expressions assumed that $f_{d} T<f_{c} T$ (for a valid computation of MSE1 as (18) and (19)) and then are valid only for $\frac{\sigma_{n}^{2}}{\sigma_{\alpha}^{2}}<\frac{1}{2}$, since with the CM criterion we get $\sigma_{e} \approx \sqrt{2} \pi f_{d} T$, and then $f_{c} T \approx K_{\infty} \approx \frac{\sigma_{e}}{\sigma_{n}} \approx \frac{\sqrt{2} \pi f_{d} T}{\sigma_{n}}$.

However, for the MAV criterion, we can find the coefficient of the AR(1) model by minimizing the MSE in (16) with respect to $a$, assuming (19) and (21):

$$
a^{*}=\sqrt{1-4 \sqrt[3]{\left(\pi f_{d} T\right)^{4} \frac{\sigma_{n}^{2}}{\sigma_{\alpha}^{2}}}}
$$

and the theoretical corresponding MSE is

$$
M S E^{*}=\frac{3}{2}\left(\pi f_{d} T \sigma_{n}^{2} \sigma_{\alpha}\right)^{\frac{2}{3}}
$$

In Fig. B.1, we compare the MSE obtained with CM and MAV criteria. We also plot the BCRB (for $k=2000$ ) as reference.

- With the CM criterion, the MSE is approximately constant with respect to the Doppler frequency and at low Doppler, the error seems to be far from the BCRB. This agrees with equations (22) and (23) since at low $f_{d} T \leq 10^{-2}$ and medium $\operatorname{SNR}$ (i.e., $\left.\frac{\sigma_{n}^{2}}{\sigma_{\alpha}^{2}} \in[0.01 ; 0.5]\right), M S E 2_{C M}$ is small and the mean square error $M S E_{C M} \approx$ $M S E 1_{C M} \approx \sigma_{n}^{2}$.

- With the MAV criterion, $M S E^{*}$ seems to be approximately the same as the MSE computed by Monte Carlo simulation for all the usual range of SNR between 0 and $20 \mathrm{~dB}$, so we validate the closed-form expression (25).

- The most important observation is that with the optimization (MAV criterion), we are closer to the bounds than with the CM criterion, especially for low frequencies $\left(f_{d} T=10^{-4}\right)$ and low SNRs (this corroborates the results of [1]).

In Fig. B.2, we complete our comparison with the AR(2) KF (under the CM criterion) of [4], and the AR(1) KF of [13] where the AR(1) coefficient $a$ is estimated on-line from observations under the assumption that the channel is a true AR(1) process (instead of a Jakes process). The performance of the first algorithm is close to that of the $\mathrm{AR}(1) \mathrm{KF}$ under the $\mathrm{CM}$ criterion (that corroborates the results in [1] and [4]) while the second one is poorer, and then the AR(1) KF optimized by the MAV criterion performs better than both of them.

Although the knowledge of $S N R=\frac{\sigma_{\alpha}^{2}}{\sigma_{n}^{2}}$ is required to design the KF in (8), its value is 
also needed for MAV-based optimization while it is not for the CM criterion. For the sake of fairness, we depict the sensitivity to imperfect SNR knowledge: Fig. B.2 plots the MSE versus the true $S N R$ for wrong values of $a$ (obtained from (24) using arbitrary values of the $\mathrm{SNR}$, fixed to $S N R^{\prime}=10,15$, or $20 \mathrm{~dB}$ ), it shows that the sensitivity to the SNR knowledge is quite weak and that MAV-based optimization even with an SNR mismatch outperforms other CM-based methods.

\section{Conclusion}

This paper addresses the problem of estimating a Rayleigh channel using a first order AR model. An analytic study clearly shows that the most widely used choice for the AR(1) pole estimation (the CM criterion) is not accurate for low SNR and low Doppler $f_{d} T$. Therefore, switching to an estimation error variance criterion as already proposed in [1], we carry out the optimization of the AR(1) model and the calculation of its performance. We provide an approximate expression of the MSE for the CM and MAV criteria first, and of the AR(1) (MAV) parameter for a given SNR and Doppler scenario. It is demonstrated that the MSE of the AR(1) KF (MAV) is proportional to the $(2 / 3)$ power of the product $\left(f_{d} T \times \sigma_{n}^{2}\right)$, where $\sigma_{n}^{2}$ is the observation noise variance.

\section{Appendix A. Approximations of $\Delta$ in (12)}

With the assumption $\sigma_{e}^{2}<<\sigma_{n}^{2}$, it is straightforward to see that

$$
\Delta=\left(\sigma_{n}^{2}+\sigma_{e}^{2}-a^{2} \sigma_{n}^{2}\right)^{2}+4 a^{2} \sigma_{n}^{2} \sigma_{e}^{2}=\sigma_{n}^{2}\left[\frac{\sigma_{n}^{2} \sigma_{e}^{4}}{\sigma_{\alpha}^{4}}+4 \sigma_{e}^{2}-4 \frac{\sigma_{e}^{4}}{\sigma_{\alpha}^{2}}\right] \approx 4 \sigma_{n}^{2} \sigma_{e}^{2}
$$

Note that for an AR(1) model with CM criterion, we have $\frac{\sigma_{e}^{2}}{\sigma_{\alpha}^{2}}=1-a_{C M}^{2}=1-\left[J_{0}\left(2 \pi f_{d} T\right)\right]^{2}=$ $1-\left[1-\left(\pi f_{d} T\right)^{2}+\frac{\left(\pi f_{d} T\right)^{4}}{2}-\ldots .\right]^{2} \approx 2\left(\pi f_{d} T\right)^{2} \leq 2 \pi^{2} 10^{-4}$ (due to the low Doppler assumption, i.e., $f_{d} T \leq 10^{-2}$ ). And due to the additional usual range SNR assumption (i.e., $0 d B \leq S N R \leq 20 d B$ and thus $0.01 \leq \frac{\sigma_{n}^{2}}{\sigma_{\alpha}^{2}} \leq 1$ ), we verify the assumption $\sigma_{e}^{2}<<\sigma_{n}^{2}$

\section{Appendix B. Approximation of $T(z)$ and Approximation of MSE1 in (18)}

The approximations made to obtain (18) are:

1. The steady-state KF, T(z), can be approximated in the frequency domain using $z^{-1}=e^{-j 2 \pi f T} \approx 1-j 2 \pi f T$ for low frequencies compared to the sampling-rate, i.e., for $f<<1 / T$, by:

$$
T\left(e^{j 2 \pi f T}\right) \approx \frac{K_{\infty}}{1-a\left(1-K_{\infty}\right)(1-j 2 \pi f T)}=\frac{A}{2 \pi f_{c} T+j 2 \pi f T}
$$

then

$$
1-T\left(e^{j 2 \pi f T}\right) \approx \frac{2 \pi f_{c} T-A+j 2 \pi f T}{2 \pi f_{c} T+j 2 \pi f T}=\frac{2 \pi f_{V L F} T+j 2 \pi f T}{2 \pi f_{c} T+j 2 \pi f T}
$$


where $A=\frac{K_{\infty}}{a\left(1-K_{\infty}\right)}, 2 \pi f_{c} T=\frac{1-a\left(1-K_{\infty}\right)}{a\left(1-K_{\infty}\right)}$, and $2 \pi f_{V L F} T=2 \pi f_{c} T-A=\frac{1-a}{a}$.

Note that for proper tracking, the cut-off frequency of the steady-state filter, $f_{c}$, should be chosen higher than the Doppler frequency $f_{d}$, but much lower than the sampling rate $1 / T$. The two conditions are quite compatible in the low Doppler scenario, and are summarized by $f_{d} T<f_{c} T<<1$.

Note also that $2 \pi f_{V L F} T=\frac{1}{a}-1$, and given $\frac{1}{a}=\left[1-\frac{\sigma_{e}^{2}}{\sigma_{\alpha}^{2}}\right]^{-\frac{1}{2}} \approx 1+\frac{1}{2} \frac{\sigma_{e}^{2}}{\sigma_{\alpha}^{2}}$, we have $2 \pi f_{V L F} T \approx \frac{1}{2} \frac{\sigma_{e}^{2}}{\sigma_{\alpha}^{2}}$. And according to Appendix A, we have for the AR(1) model with $\mathrm{CM}$ criterion, $\frac{\sigma_{e}^{2}}{\sigma_{\alpha}^{2}} \approx 2\left(\pi f_{d} T\right)^{2}<<4 \pi f_{d} T$. From now on, our complete assumptions for the computation of the integral (17) will be:

$$
f_{V L F} T<<f_{d} T<f_{c} T<<1
$$

2. The function $1-T\left(e^{j 2 \pi f T}\right)$ can be (asymptotically) divided into three parts (as can be checked by the plot of $\left|1-T\left(e^{j 2 \pi f T}\right)\right|^{2}$ in Fig. B.3):

(a) for $0<f T<f_{V L F} T$, a constant part approximately equal to $\frac{2 \pi f_{V L F} T}{2 \pi f_{c} T}$

(b) for $f_{V L F} T<f T<f_{c} T$, a linear part approximately equal to $\frac{2 \pi f T}{2 \pi f_{c} T}$

(c) for $f T>f_{c} T$, a constant part equal to 1, not considered in the integral (17), since $\Gamma_{\alpha}(f)=0$ for $|f|>f_{d}$, with $f_{d} T<f_{c} T$.

Then

$$
\begin{aligned}
M S E 1 & \approx 2 \int_{0}^{+f_{V L F}}\left[\frac{2 \pi f_{V L F} T}{2 \pi f_{c} T}\right]^{2} \Gamma_{\alpha}(f) d f+2 \int_{+f_{V L F}}^{+f_{d}}\left[\frac{2 \pi f T}{2 \pi f_{c} T}\right]^{2} \Gamma_{\alpha}(f) d f(\mathrm{~B} .2) \\
& =2 I_{1}\left[0 ;+f_{V L F}\right]+2 I_{2}\left[+f_{V L F} ; f_{d}\right]
\end{aligned}
$$

but $I_{2}\left[0 ;+f_{V L F}\right]<I_{1}\left[0 ;+f_{V L F}\right]$ since $f<f_{V L F}$ in this computation, and $I_{2}\left[+f_{V L F} ;+f_{d}\right]$ $<I_{2}\left[0 ;+f_{d}\right]$, then

$$
2 I_{2}\left[0 ;+f_{d}\right]<M S E 1<2 I_{1}\left[0 ;+f_{V L F}\right]+2 I_{2}\left[0 ;+f_{d}\right]
$$

but given the form of $\Gamma_{\alpha}$, it results that $I_{1}\left[0 ;+f_{V L F}\right]<\frac{f_{V L F}}{f_{d}} I_{2}\left[0 ;+f_{d}\right]<<I_{2}\left[0 ;+f_{d}\right]$ since $f_{V L F}<<f_{d}$.

In conclusion, $M S E 1 \approx 2 I_{2}\left[0 ;+f_{d}\right]=2 \int_{0}^{+f_{d}}\left[\frac{2 \pi f T}{2 \pi f_{c} T}\right]^{2} \Gamma_{\alpha}(f) d f=\frac{\left(2 \pi f_{d} T\right)^{2}}{2\left(2 \pi f_{c} T\right)^{2}} \sigma_{\alpha}^{2}$.

\section{References}

[1] A. Barbieri, A. Piemontese, G. Colavolpe, "On the ARMA Approximation for Frequency Channels Described by the Clarke Model with Applications to Kalmanbased Receivers", in IEEE Trans. on Wireless Comm., vol. 8, no. 2, Feb. 2009.

[2] K. E. Baddour and N. C. Beaulieu, "Autoregressive modeling for fading channel simulation", in IEEE Trans. Wireless Comm., vol. 4, no. 4, pp. 1650-1662, Jul. 2005. 
[3] H. S. Wang and P. C. Chang, "On verifying the first-order Markovian Assumption for a Rayleigh Fading Channel Model”, in IEEE Trans. on Vehicular Tech., vol. 45, no. 2, pp. 353-357, May 1996.

[4] C. Komninakis, C. Fragouli, A. H. Sayed and R. D. Wesel, "Multi-Input MultiOutput fading channel tracking and equalization using Kalman estimation", in IEEE Trans. on Signal Proc., vol. 50, May 2002.

[5] Z. Liu, X. Ma, and G. B. Giannakis, "Space-time coding and Kalman Filtering for time selective fading channels", in IEEE Trans. on Comm., vol. 50, no. 2, Feb. 2002.

[6] M. McGuire and M. Sima, "Low-order Kalman filters for channel estimation", in IEEE Comm., Computers and Signal Proc., pp. 352-355, Aug. 2005.

[7] W. Chen and R. Zhang, "Kalman-filter channel estimator for OFDM systems in time and frequency-selective fading environment", in Proc. IEEE ICASSP, Montréal, Canada, May 2004.

[8] T. Y. Al-Naffouri, "An EM-based forward-backward Kalman for the estimation of time-variant channels in OFDM", in IEEE Trans. on Signal Proc., vol. 55, Jul. 2007

[9] H. Hijazi and L. Ros, "Joint Data QR-Detection and Kalman Estimation for OFDM Time-varying Rayleigh Channel Complex Gains", in IEEE Trans. Comm., vol. 58, no. 1, pp. 170-178, January 2010.

[10] H. Abeida, J.-M. Brossier, L. Ros and J. Vilà-Valls, "An EM algorithm for path delay and complex gain estimation of a slowly varying fading channel for CPM signals", in Proc. IEEE Globecom, Hawaï, Dec. 2009.

[11] L. Ros, H. Hijazi, E. P. Simon, "Paths complex gain tracking algorithms for OFDM receiver in slowly-varying channels", in Proc. IEEE ISCCSP, Limassol, Cyprus, Mar. 2010.

[12] H. Hijazi and L. Ros, "Bayesian Cramér-Rao Bounds for complex gain parameters estimation of slowly varying Rayleigh channel in OFDM systems" in ELSEVIER Signal Processing Fast Communication, vol. 89, pp. 111-115, Jan. 2009.

[13] M. Enescu and V. Koivunen, "On the estimation of state matrix and noise statistics in state space models", in Proc. IEEE 56th Vehicular Technology Conference, Vancouver BC, Sep. 2002.

[14] G. K. Chui and G. Chen, Kalman Filtering with real-time applications, SpringerVerlag, 1987.

[15] S. Ghandour, Estimation de canal à évanouissements lents pour les communications radio-mobiles, Master's degree report at Grenoble-INP, Jun. 2010.

[16] Steven M. Kay, Fundamentals of Statistical Signal Processing - Estimation Theory. Prentice Hall, 1993. 


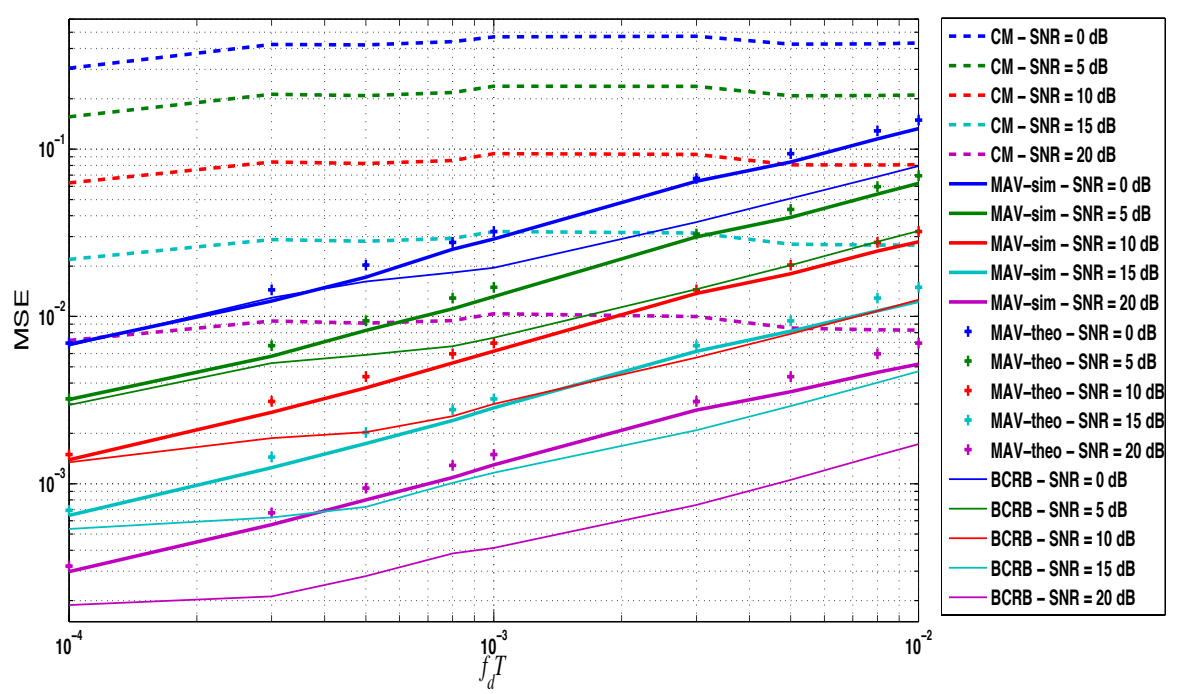

Figure B.1: MSE comparison of the AR(1) KF estimator based on the literature CM criterion (7) or on the $M A V$ criterion (24), for different $S N R$, as functions of $f_{d} T$.

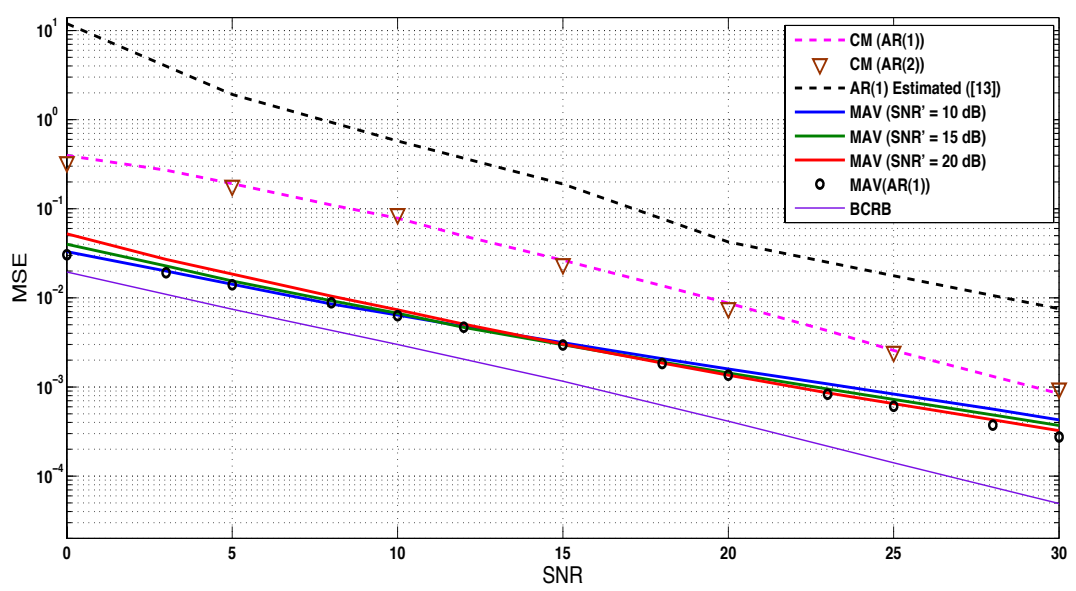

Figure B.2: $\quad$ MSE comparison versus $S N R$ for $f_{d} T=5 \cdot 10^{-4}$ of the AR(1) KF (MAV and CM), AR(2) KF $(C M)$, and $A R(1) K F$ of [13], and the effect of a mismatch on the knowledge of the SNR for the AR(1) KF $(M A V)$ with an $A R(1)$ coefficient a computed by (24) with (false) fixed values of $S N R, S N R^{\prime}=10,15$ and $20 \mathrm{~dB}$ (solid lines), and (true) SNR (circles).

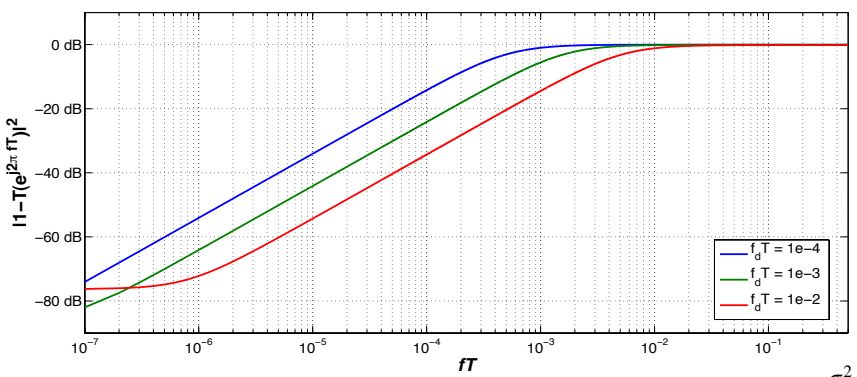

Figure B.3: The high-pass filter $1-T(z)$ for various $f_{d} T, S N R=20 d B$, and $\frac{\sigma_{e}^{2}}{\sigma_{\alpha}^{2}}=\frac{f_{d} T}{1000}$ 